\title{
Letrozole Therapy in Cases of Endometrial Hyperplasia Not Responding to Gestagen Therapy
}

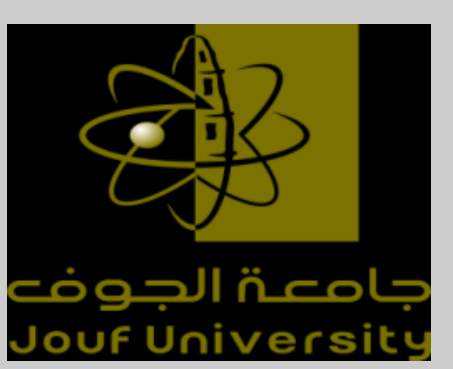

\section{Ibrahim Elbahlool 1,2*, Tarek Shokeir ${ }^{2}$, Elkhiary Mostafa ${ }^{2}$, Dina Elsaid ${ }^{2}$ \\ 1 Obs \& Gyn department- College of Medicine, Jouf university .KSA. 2 Obs \& Gyn department- College of Medicine, Mansoura university .Egypt.}

Introduction: Endometrial hyperplasia is a condition of excessive proliferation of the cells of the endometrium which may present with abnormal uterine bleeding and considered an important risk factor for the development or even co-existence of endometrial cancer. Geatagen failure can be defined as progression to atypical hyperplasia that occurs during follow-up, no histological regression of hyperplasia despite 12 months of treatment, relapse of endometrial hyperplasia after completing hormonal treatment, or Persistent bleeding.

Aim : The aim of this trial was to evaluate the efficacy of letrozole as a treatment for endometrial hyperplasia without atypia not responding to gestagen therapy and to study the outcome of two doses different regimens.

Patients and methods: This prospective randomized study was conducted at Mansoura University Hospital, department of Obstetrics \& Gynaecology. A total of 46 women diagnosed with simple endometrial hyperplasia without atypia with failed gestagen therapy (for at least one year) were enrolled. History taking and examination was done to all cases. Eligible participant subjects who met the inclusion and exclusion criteria were randomly assigned to either: Group A included 20 patients who received letrozole (Letrozole Tablets, Synthon Pharmaceuticals, Inc.) in a dose of 2.5 mg daily for 3 months by non-stop regimen. Group B included 20 patients who received letrozole; in a dose of $5 \mathrm{mg}$ daily $(2.5 \mathrm{mg}$ twice daily) for 3 months by non-stop regimen .

\section{$\underline{\text { Results }}$}

Table 1: Patients' characteristics and demographic data in both groups

\begin{tabular}{|c|c|c|c|}
\hline & $\begin{array}{c}\text { Group(A) } \\
\text { Letrozole } 2.5 \mathrm{mg} \\
\text { (19 patient) }\end{array}$ & $\begin{array}{c}\text { Group(B) } \\
\text { Letrozole 5mg } \\
\text { (18 patients) }\end{array}$ & $P$ value \\
\hline$\overline{\text { Age (mean } \pm \text { SD) }}$ & $55.7 \pm 10.57$ & $55.2 \pm 10.83$ & $\begin{array}{c}0.896= \\
\text { NS }\end{array}$ \\
\hline BMI (mean \pm SD) & $36.9 \pm 4.2$ & $35.7 \pm 3.97$ & $\begin{array}{c}0.389= \\
\text { NS }\end{array}$ \\
\hline $\begin{array}{l}\text { Parity (median and range) } \\
\text { Medical problems }\{\mathrm{n}(\%)\} \text { : }\end{array}$ & 3 with range 9 & 3 with range 8 & NS \\
\hline 1. DM & $8(42.1 \%)$ & $7(38.9 \%)$ & $0.842=$ \\
\hline 2. HTN & $10(52.6 \%)$ & $9(50 \%)$ & $\begin{array}{c}\text { NS } \\
0.873= \\
\text { NS }\end{array}$ \\
\hline \multicolumn{4}{|l|}{ Menopausal state $\{\mathrm{n}(\%)\}$ : } \\
\hline 1.premenopausal & $6(31.6 \%)$ & $5(27.8 \%)$ & \multirow{2}{*}{$\begin{array}{c}0.800= \\
\text { NS }\end{array}$} \\
\hline 2.postmenopausal & $13(68.4 \%)$ & $13(72.2 \%)$ & \\
\hline $\begin{array}{l}\text { Presenting symptoms }\{\mathrm{n} \\
(\%)\}:\end{array}$ & $13(68.4 \%)$ & $13(72.2 \%)$ & \multirow{4}{*}{$\begin{array}{c}0.503= \\
\text { NS }\end{array}$} \\
\hline 1.postmenopausal bleeding & $6(31.6 \%)$ & $4(22.2 \%)$ & \\
\hline 2.intermenstrual bleeding & & & \\
\hline $\begin{array}{l}\text { 3.regular excessive bleeding } \\
\text { Method of initial diagnosis } \\
\text { \{n (\%)\}: }\end{array}$ & & & \\
\hline 1.inpatient (D\&C) & $10(52.6 \%)$ & $5(27.8 \%)$ & \multirow{3}{*}{$\begin{array}{c}0.124= \\
\text { NS }\end{array}$} \\
\hline 2.outpatient (Novac) & $9(47.4 \%)$ & $13(72.2 \%)$ & \\
\hline Type of EH: & & & \\
\hline 1.simple & $19(100 \%)$ & $17(94.4 \%)$ & \multirow{2}{*}{$\begin{array}{c}0.298= \\
\text { NS }\end{array}$} \\
\hline 2.complex & 0 & $1(5.6 \%)$ & \\
\hline
\end{tabular}

Table 2: Endometrial thickness measured by TVS

\begin{tabular}{|l|l|l|l|l|}
\hline & Baseline & $\begin{array}{l}\text { After 1 } \\
\text { month }\end{array}$ & $\begin{array}{l}\text { After2 } \\
\text { months }\end{array}$ & $\begin{array}{l}\text { After 3 } \\
\text { months }\end{array}$ \\
\hline $\begin{array}{l}\text { Group(A) Letrozole } \mathbf{2 . 5} \mathbf{~ m g} \\
(\mathbf{n}=\mathbf{1 9})\end{array}$ & $13.5 \pm 3.6$ & $10.1 \pm 2.8$ & $6.4 \pm 2.1$ & $\begin{array}{l}4.4 \pm \\
1.95\end{array}$ \\
\hline $\begin{array}{l}\text { Group(B) Letrozole } \mathbf{5 m g} \\
(\mathbf{n}=\mathbf{1 8})\end{array}$ & $13.2 \pm 3.4$ & $9.8 \pm 2.7$ & $6.5 \pm 2.4$ & $4.8 \pm 1.5$ \\
\hline P value & 0.79 & 0.73 & 0.89 & 0.48 \\
\hline
\end{tabular}

Table 3: Hysteroscopic re-assessment and endometrial biopsy rates

\begin{tabular}{l|llll|} 
& Baseline & $\begin{array}{l}\text { After 1 } \\
\text { month }\end{array}$ & $\begin{array}{l}\text { After2 } \\
\text { months }\end{array}$ & $\begin{array}{l}\text { After 3 } \\
\text { months }\end{array}$ \\
\hline $\begin{array}{l}\text { Group(A) Letrozole 2.5 mg } \\
(\mathbf{n = 1 9 )}\end{array}$ & $19(100 \%)$ & $2(10.5 \%)$ & 0 & $2(10.5 \%)$ \\
$\begin{array}{l}\text { Group(B) Letrozole } 5 \mathrm{mg} \\
(\mathbf{n = 1 8})\end{array}$ & $18(100 \%)$ & 0 & $1(5.55 \%)$ & $2(11.11 \%)$ \\
P value & NS & 0.16 & 0.30 & 0.95
\end{tabular}

Table 4: Outcome of both groups according to the results of endometrial histopathology obtained 3 months' post treatment

\begin{tabular}{|l|l|l|}
\hline & Resolution & Persistence \\
\hline Group(A) Letrozole $2.5 \mathrm{mg} \mathrm{(n=19)}$ & $17(89.5 \%)$ & $2(10.5 \%)$ \\
\hline Group(B) Letrozole $5 \mathrm{mg}(\mathbf{n = 1 8})$ & $17(94.4 \%)$ & $1(5.55 \%)$ \\
\hline P value & 0.33 & 0.59 \\
\hline
\end{tabular}

Table 5: Failure rate

\begin{tabular}{|c|c|c|c|}
\hline Variable & $\begin{array}{l}\text { Group (A) Letrozole 2.5 } \\
\text { mg (n=19) }\end{array}$ & $\begin{array}{l}\text { Group(B) Letrozole } \\
\text { 5mg (n=18) }\end{array}$ & $\begin{array}{l}\mathbf{P} \\
\text { value }\end{array}$ \\
\hline Progression & 0 & 0 & 0.5 \\
\hline $\begin{array}{c}\text { No regression } \\
\text { (persistence) }\end{array}$ & $2(10.5 \%)$ & $1(5.5 \%)$ & 0.29 \\
\hline Renewed bleeding & $2(10.5 \%)$ & $2(11.1 \%)$ & 0.48 \\
\hline
\end{tabular}

Data are expressed as $\{n(\%)$.

\section{Conclusion}

Patients with simple endometrial hyperplasia without atypia with failed Gestagen therapy can be managed successfully with Letrozole. Both dose regimens used succeeded in management of simple endometrial hyperplasia without atypia with no significant difference between them as regard the clinical, radiological or histological outcome. However, the second dose group B (5 mg) was associated with higher side effects, failure rates, cost and less patient compliance, so, group A $(2.5 \mathrm{mg})$ is preferred, however further RCT are warranted to prove or disprove this. 